\title{
El Factor Ginecológico en el Estudio de la Esterilidad
}

Doctor Héctor Enrigue Bernal B. (Profeser Agregado de Ginecologia de la Universidad Nacional. Adjunto del Departamento de
Ginecologia del Hospital de San José).

Terminado el interrogatorio entramos al examen clínico propiamente dicho in el cual debemos considerar ante todo el aspecto general, para estudiar luégo d) aparato especializado de la reproducción.

El examen general, previa la determinación de algunos valores antropométricos indispensables como talla, peso, envergadura, etc., debe comenzar por una inspección cuidadosa, que algunas veres nos revela datos muy interesantes. Con bastante insistencia se habla de la relación esterilidad: biotipo femenino: según algunos autores el normotipo sería fecundo, lo serían menos en orden decreciente el tipo juvenil, el tipo asténico-ptósico, el hipoplástico y el tipo intersexual Pero. como lo dice Stabile, si bien esto debe admitirse como hecho de observaión clínica, lo lógico es considerar a la esterilidad de tipo constitucional entre us factores de origen endocrino ya que "al fin y al cabo las alteraciones del biotipo son función casi exclusiva del equilibrio endocrino".

La escuela americana considera las endocrinopatías como causantes de muchos casos de infertilidad femenina, lo cual es un hecho perfectamente establecido. Sin embargo con alguna frecuencia se observa que trastornos endocrinos severos como una insuficiencia tiroidea, una diabetes mellitus u otros síndromes aún más complejos no estan invariablemente asociados con la esterilidad e inversamente que muchos casos de infertilidad en los que no se encuentra causa local, no están asociados tampoco con trastornos endocrinos o constitucionales. Vo es este el lugar para extendernos sobre el tema que por lo demás será detaHado en el capitulo correspondiente; tan sólo queremos llamar la atención sobre la importancia de una cuidadosa inspección que comenzando por el biotipo se detenga en los caracteres sexuales secundarios, estudie la conformación de los senos, la distribución de grasa y del sistema piloso y descubra los posibles estigmas en- 
docrinos: si alguna anormalidad se observare, ella debe ser investigada a fondo y valorada entre los posibles factores de la esterilidad sin pretender concluír que sea la causa única.

A la inspección general debe seguir el examen clínico metódico de los diversos órganos y aparatos. Ya mencionábamos, a propósito del interrogatorio, la im portancia muy grande que algunas afecciones como la apendicitis, las enfermedades inflamatorias de la pelvis o las enfermedades venéreas pueden tener en la génesis de la esterilidad. Mucho menor importancia se concede a otras condiciones que, como la pielitis crónica, la sinusitis, las infecciones apicales de los dientes y la toxemia crónica de origen intestinal, para no mencionar sino las más frecuentes, pueden también prevenir la concepción sea temporalmente o de una manera definitiva. Ellas obran de varias maneras: con frecuencia afec$\tan$ la buena penetrabilidad del moco cervical; en otros casos reducen o inva lidan la respuesta del aparato genital a las hormonas, como sucede en algunas mujeres afectadas de pielitis crónica en quienes a pesar de las grandes dosis no se logran provocar los efectos específicos de la progesterona. No es raro que tales condiciones se asocien con ciclos anovulatorios o con la viabilidad deficiente del huevo fecundado.

La investigación clinica de estos factores no específicos de infertilidad está entorpecida por dos dificultades. En primer lugar muchas de las afecciones que efectivamente previenen la concepción son sub-clínicas, en el sentido de que la paciente conserva un estado razonable de salud y no tiene síntomas; por tal motivo pone obstáculo a investigaciones que según su sentir no tienen ninguna relación con su dificultad primaria. Por otra parte las diferencias individuales complican la evaluación de los hallazgos clínicós ya que algunas mujeres conciben y llevan su embarazo felizmente en condiciones que para otras son causa de esterilidad. Pocas mujeres por encima de los 30 años, quienes por otra parte constitutyen la mayoría de las pacientes estériles, están libres de estigmas subclínicos; y puesto que cada caso no puede ser investigado de una manera completa es por lo menos indispensable eliminar los factores más groseros antes de seguir adelante. Uno de los más comunes en la práctica es la infección del tracto urinario que con frecuencia se propaga a la superficie cervical y al endocérvix; su agente causal, el colibacilo ha sido acusado por numerosos autores de prevenir la concepción en unos casos y en otros de provocar el aborto habitual. Interminables nos haríamos si intentáramos pasar revista de las afecciones extragenitales que por una causa cualquiera pueden estar asociadas con estados de infertilidad; nos limitaremos únicamente a mencionar cuatro grandes grupos:

10-La presencia de focos sépticos crónicos.

2 - Las enfermedades crónicas graves o consuntivas.

30-Ciertos trastornos dietéticos, especialmente las dietas pobres en proteinas. 4 - Los estados de agotamiento físico o psíquico.

En realidad, como lo decíamos en la primera parte, ellas no obran directa sino indirectamente a traves de las modificaciones que imprimen al medio in- 
terno, las cuales repercuten desfavorablemente en la gonada y en el funcionamiento del resto del aparato genital.

Terminado el examen clínico general nos corresponde ahora el estudio detenido del aparato genital femenino en el cual debemos considerar dos partes: $1 \%$ el aspecto somático o examen ginecológico propiamente dicho y $2^{\circ}$ el aspecto funcional. Solamente al primero habremos de referimos aquí va que el segundo constituirá el tema especializado de diversos capítulos en este estudio.

Antes de entrar en materia queremos recordar la importancia de los antecedentes ginecológicos estudiados en la primera parte: una historia menstrual $\because$ sexual completa nos proporciona, en la mayoría de las veces, las bases para mejor orientar nuestras investigaciones posteriores. Debemos también llevar en mente que por el examen ginecológico tan sólo vamos a descubrir los grandes trastornos y no todos ellos, ya que aún el más cuidadoso examen clínico no puede proporcionarnos información completa acerca de las condiciones funcionales Jel aparato reproductor, vale decir por ejemplo del endometrio cuyo desarroIlo puede ser incompleto o que no reacciona favorablemente a los estimulos provenientes del ovario. Por último no debemos olvidar la posibilidad de que en un caso dado puedan concurrir muchos factores en la reducción de la fertilidad.

Siguiendo a Lane-Roberts consideraremos cuatro grandes grupos de afecciones ginecologicas que pueden afectar la fertilidad.

I. Grupo: Infección vulvo-vaginal, cervical o uterina.

En realidad las afecciones comprendidas en este grupo no son causas for zosas de esterilidad, pero deben ser incluídas entre los factores coadyuvantes: por consiguiente habremos de prestarles particular atención. No entramos en detalles sobre la manera de reconocer una bartholinitis, una infección de las Glandulas de Skerre o una uretritis, ni tampoco sobre el conocido mecanismo cie autodepuracion vaginal y la manera como se perturba; bástenos recordar que en la génesis de todo flujo vaginal primitivo existen causas endógenas o xćgenas y que es necesario investigar no sólo el posible agente específico sino - ambien las variaciones de la acidez normal de la vagina. Está demostrado que 1 flujo vaginal produce una intensa inhibición en los movimientos de los espermatozoides, cuya causa radica probablemente en la formacion anormal de toxinas microbianas en la vagina: en general, cuanto menor es la pureza del contenido vaginal menor es la probabilidad de fecundación. No olvidemos tampoco que en muchísimos casos la alteración del medio vaginal no es sinı el reflejo de una alteración en el funcionamiento del ovario y que por consiquiente es en este último en donde debemos buscar la verdadera causa de la esterilidad.

Por razones semejantes a las aducidas a propósito de las vaginitis comprenderemos que la fecundación está profundamente obstaculizada en el curso de una endometritis aguda. Problema diferente es el de la infección crónica del endometrio que según los conceptos modernos, excepción hecha de la infec- 
ción tuberculosa o sifilítica, no puede persistir en razón de la renovación periódica de la funcional del epitelio endouterino. Sin embargo se olvida que la in fección puede permanecer en forma latente en la profundidad de la basal, mánifestándose acasionalmente en forma de alteraciones menstruales asociadas con sensibilidad local. En estas condiciones es perfectamente posible la nidación del huevo fecundado más no siempre su ulterior desarrollo: al sufrir la mucosa su transformación en caduca se desarrolla una endometritis decidual que clínicamente se caracteriza por hidrorrea y metrorragias y finalmente por el aborto. Nuevas tecundaciones pueden producirse con igual resultado: tal ta génesis de muchos casos de aborto habitual. Como lo dice Stabile: "es digno de tenerse presente el hecho de que en muchos de estos casos, fuera de la gestación, la endometritis no da lugar a síntomas clínicos, debido a que la capa funcional poco afectada responde normalmente a los estímulos provenientes del ovario". Recientemente algunos autores, teniendo en cuenta la existencia de estas endometritis latentes, han comenzado a emplear cultivos de endometrio con el objeto de descubrir presuntas infecciones responsables de infecundidad o de aborto habitual.

Hemos dejado para última en este grupo a la infección cervical, con el objeto de resaltar su importancia, porque si en riguroso análisis ella no está necesariamente asociada con la infertilidad, sin embargo en muchos casos no se produce la concepción mientras no se la trata de una manera efectiva; de ahí que hoy se le considere como una de las causas más comunes de esterilidad femenina. Su estudio detallado se hará a propósito del factor cervical.

II Grupo: Malformaciones e hipoplasia.

Mencionaremos solamente las más importantes. Algunas de ellas como las vaginales y vulvares tienen gran interés desde el punto de vista de la copulación, ya que al impedir o dificultar la inmisión o penetración del miembro viril en la vagina se oponen a la inseminación directa del cuello uterino. Tal sucede por ejemplo con el himen imperforado y la rigidez invencible del mismo, aunque el primero, en razón de los trastornos que provoca en la época de la pubertad, es generalmente reconocido y tratado antes del matrimonio; la aplasia parcial y las estenosis de la vagina, estas últimas con frecuencia adquiridas; por último, diversos grados de detención en el desarrollo de vúlva y vagina, generalmente asociados a vicios semejantes en útero y anexos, y que van desde la hipoplasia uterina hasta la ausencia completa de una o más estructuras histológicas.

El útero hipoplásico presenta con frecuencia un marcado grado de anteflexión y su tamaño es a veces menor que el de una nuez; sin embargo Meaker ha demostrado que, como índice de madurez uterina, tiene muchísimo mayor valor la relación cuerpo-cuello que el tamaño. El útero inmaduro está caracterizado por la longitud relativamente grande del cuello comparada con la del cuerpo, proporción que tiende a invertirse a medida que prosigue el desarrollo hacia la madurez, así, en el útero normalmente desarrollado, en la época de la madurez sexual, la relación del cuerpo con el cuello es de $2 / 1$, en tanto que en el útero infantil es de $1 / 2$ y en el útero puberal de $1 / 1$. Si con ayuda de un histe- 
rómetro determinamos la longitud total del útero y le restamos la parte correspondiente a la cavidad cervical tendremos la longitud del cuerpo, la cual dividida por la del cuello nos dará un cuociente; ahora bien el resultado de dividir este cuociente por dos es lo que se conce con el nombre de indice uterino, cuva interpretación, de acuerdo.con Meaker, es como sigue:

Cifras de 0,75 o mayores indican útero adulto normal.

Cifras de 0,60 o menores indican hipoplasia digna de tenerse en cuenta desde el punto de vista clínico; y, por último,

Cifras de 0,25 o vecinas son indice de un útero completamente infantil.

Debemos recordar que aún cuando la hipoplasia no significa de una manera absoluta disminución de la fertilidad, constituye sin embargo uno de sus signos más comúnes, pudiendo llegar, cuando es muy acentuada, a representar factor importantísimo de esterilidad, de la cual en realidad es un síntoma dentro del cuadro más complejo de la insuficiencia ovárica.

Mencionemos por último algunas anormalidades referentes al cuello como lá flexión exagerada a la altura del itsmo, el alargamiento, la hipertrofia o el descenso. El cuello largo y cónico, incurvado y con orificio puniforme con mucha frecuencia está asociado a la infertilidad, en razón de la insuficiente cantidad de moco generalmente tan espeso que lo hace impenetrable a los espermatozoides: este punto será ampliamente desarrollado a propósito del factor cervical.

III Grupo. Vicios de posición. - Deformaciones y tumores.

El concepto sobre el significado de la posición uterina ha cambiado en los últimos años: en la actualidad se admite generalmente que la retroversićn uterina no constituye de por sí un factor decisivo de esterilidad, pues la mayoría de las mujeres portadoras de este vicio de posición conciben con toda facilidad. De existir, la esterilidad es debida, en parte, en estos casos a la hipoplasia sexual concomitante y en parte a la desviación hacia adelante del orificio externo del cuello que, al quedar tapado por la pared anterior de la vagina, escapa a la inseminación directa; no deja de ser significativo que el Test post-coital se vuelva positivo. después de haber sido sistemáticamente negativo, cuando se ha corregido la malposición uterina. En la misma forma un vicio de posicion, de por sí anodino, puede ser causa de infertilidad cuando determina una torsión en la luz de la trompa.

En la evaluación del significado de una malposición es necesario tener en cuenta los signos y síntomas asociados: por.ejemplo un desplazamiento móvil es menos probable que esté asociado a la infertilidad que uno fijo, ya que este último es con frecuencia causado por adherencias consecutivas a una afección inflamatoria. Del mismo modo debemos prestar atención a la sensibilidad $y$ a los anexos prolabados en razón dé sus relaciones con la dispareunia: en algunos casos la sensibilidad es tal, que la posición y movilidad del útero pueden definirse únicamente bajo anestesia.

El hallazgo de una latero-desviación sugiere la presencia de anormalidades en los anexos, ya sean de origen tumoral o inflamatorio; menor es el significado de otras condiciones como el prolapso y la hiperflexión congénita con estenosis 
del orificio interno del cuello ya que como lo hacen notar algunos autores, a propósito de la última, "el calibre del itsmo uterino nunca es menor al del orificio uterino de las trompas, que los espermatozoides franquean sin embargo con facilidad" (Ahumada).

Al hablar de las deformaciones uterinas surge el problema del mioma como factor de esterilidad, en realidad de difícil solución, como lo demuestra el hecho de que numerosas miomatosas son normalmente fecundas, en tanto que en otros casos la simple extirpación del o de los núcleos miomatosos pueden dar fin a una esterilidad debidamente comprobada. Mucho se ha debatido sobre la relación entre los dos fenómenos, como derivados ambos de un mismo prozeso de perturbación de la función ovárica; pero aparte de ello no cabe duda de que en otras oportunidades el núcleo intramural o submucoso en juego constituve el factor principal en la infertilidad. Por dicha razón se puede admitir teóricamente que un mioma que alarga y deforma la cavidad uterina, que comprime o desplaza los orificios uterinos de ambas trompas o que provoca ciertas modificaciones del endometrio (edema, congestión, ulceración, etc.), puede dificultar la ascensión de los espermatozoides, o la nidación del huevo fecundado, y en muchas ocasiones ser causa de aborto de repetición.

La importancia de los demás tumores uterinos (carcinoma del cuello principalmente) debe considerarse como puramente circunstancial.

Il Grupo: Enfermedades inflamatorias de la pelvis

Ya decíamos en la primera parte cómo la inflamación pélvica constituye una de las causas más comunes de esterilidad en la mujer; por consiguiente, el examen ginecologico debe ser especialmente cuidadoso en relación con las trompas y ovarios. A grandes rasgos recordaremos que las enfermedades inflamatorias pueden manifestarse por masas tubo-ováricas, sensibilidad en los fondos de saco laterales, engrosamiento de las bases de los ligamentos anchos (parametritis), retroflexion fija del útero, dolor provocado con los morimientos laterales del cue. llo, etc. En caso de duda debe emplearse anestesia. En cuanto sea posible debe diferenciarse la infección tubaria de la parametritis y de otras afecciones patológicas de la pelvis. La presencia de salpingo-ooforitis aguda o subaguda contraindica ulteriores procedimientos de diagnćstico, como la persuflacion tubárica.

La tuberculosis endometrial es considerada clásicamente como una enfermedad rara: sin embargo trabajos recientes han demostrado que es más común. de lo que ordinariamente se aprecia y que cuando se encuentra en el endometrio existe también en las trompas. Para no mencionar sino dos casos recordemos la estadistica de Lane-Roberts quien encontró tuberculosis endometrial no sospechada en 94 , sobre 1.712 casos no seleccionados, de esterilidad primaria, lo que equivale a 5,5 por ciento; datos semejantes ofrece la estadística de Rabau (1945) en que, sobre 1.024 casos de esterilidad, 52 presentaban tuberculosis del endometrio, es decir un índice del 5 por ciento.

Entre nosotros, aun cuando no propiamente en estudios de esterilidad, Amaya León encuentra sobre 2.186 piezas histológicas de ginecología 53 casos de 
tuberculosis genital, es decir 2,4 por ciento; y sobre 40 biópsias de endometrio, practicadas a enfermas con tuberculosis pulmonar, en dos se encontró también tuberculosis endometrial, lo que da una incidencia del 5 por ciento.

Termina aquí el examen clínico general y en particular el examen gineco lógico; pero para que los hallazgos proporcionados por la clínica alcancen su mayor valor es necesario adicionarlos y relacionarlos con los obtenidos por el laboratrio, entre los cuales hay algunos que como los de orina, materia fecal, secreciones genitales, cuadro hemático y eritrosedimentación, serología, azohemia y glicemia, metabiolismo basal, etc., son absolutamente indispensables.

Si como resultado del estudio anterior encontráramos anormalidades o lesiones claramente definidas, debemos considerarlas como posibles causas de infertilidad y tratarlas en cuanto sea posible; pero no debemos cometer el error de atribuírles la causa única de una gran esterilidad, salvo, y esto con reservas, cuando se trata de grandes anormalidades, como una hipoplasia extrema o una infección demasiado severa. Aquí, como siempre, el criterio clínico habrá de orientarnos sobre si se debe o no proseguir la investigación, decisión que no siempre es fácil. En caso afirmativo nos corresponde ahora el estudio de la función, en cuanto a reproducción se refiere, lo que resumido en cortas líneas equivale a preguntar: si el ovario ovula y si el ovulo pasa al útero, si el endometrio sufre su transformación fisiológica periódica, si la inseminación se efectúa en forma apropiada y si los espermatozoides pasan al cuello, si las condiciones del canal cervical favorecen la invasión, si el estado endocrino de la paciente y las condiciones necesarias al desarrollo del huevo se mantienen a un nivel adecuado; son estos los temas que serán desarrollados en los próximos capítulos.

\section{B I B L I O G R A F I A}

Ahumada Juan C.: "Tratado Elemental de Ginecología". Tercera Edición, Buenos Aires, 1952.

Amaya León Hernando: "Tuberculosis en Ginecologia". Revista Colombiana de Obstetricia y Ginecología. Vol. I, No 6, octubre-noviembre, 1950.

Bernal Héctor Enrique: "Miomatosis y Embarazo". "Cirugía Conservadora". Repertorio de Medicina y Cirugía. - Bogotá, Vol. V, No 2, enero-febrero, 1950.

Buxton C. L. and Mathews C. S.: "Bacteriology of the Cervix in cases of Infertility". Fertility and Sterility. Vol. 2, No 1. Jan., 1951.

Calatroni Carlos J. y Ruiz Vicente: "Terapéutica Ginecológica". III Edición, "El Ateneo", Buenos Aires, 1944. 
Conill M. Victor : "Tratado de Ginecología". Barcelona, 1946.

Cotte Gastón: "Troubles Fonctionnels de L'Appareil Genital de la Femme". Masson et Cie. París, 1931.

Hamblen E. C.: "Endocrinology of Woman". Charles C. Thomas. Springfield III, 1950.

Curtis Arthur Hale: "Cinecologia”. Barcelona, 1941.

Jensen R. M. and McDonald J. R.: Am. J. Obst-Gynec. Vol. 41, Pág. 268, 1941.

Lane-Roberts Cedric: "Sterility and Impaired Fertility". Paul B. Hoeber Inc. Second Edition. London, 1948.

Meaker S. R.: "Human Sterility". Baltimore, Williams and Wilkins Co., 1934.

Notas Médicas (Schering Corporation). Vol. II, No 9. Sept. 1948.

Stabile Américo: "La Esterilidad Matrimonial". Buenos Aires "El Ateneo", 1947.

Taylor Howard C. Jr.: "Vascular Congestion and Hyperemia". Am. J. of Obst-Gynec. Vol. 57, Numbers 2 and 4, 1949.

Taylor Howard C. Jr.: "Life Situations, Emotions and Gynecologic pain Associated with Congestion". 